\title{
Distortion of parallel lines in geometrical fields as a function of size of the display
}

\author{
A. J. MARSHALL AND VINCENT DI LOLLO \\ UNIVERSITY OF WESTERN AUSTRALIA
}

The illusory distortion of two parallel lines on the Hering, Wundt, and Orbison fields was studied as a function of the size of the display. The Hering and Wundt fields yielded complementary illusions which developed with the size of the display. The Orbison field yielded a lesser amount of illusion and different development.

Marshall \& Di Lollo (1963) measured the extent of Hering illusion under comparable degrees of impoverishment in photopic and scotopic vision. The normal illusion was absent at high levels of impoverishment and developed steadily with improvement in viewing conditions. At small visual angles in photopic viewing, a reversal of the normal illusion was obtained, in which the "parallel" lines appeared closer together at the center of the figure. These results were interpreted in terms of local effects of intersecting lines on the magnitude and direction of the illusion.

The present study compares the development of illusory distortion of a pair of parallel lines when set in each of three geometrical fields as a function of the size of the display at a low level of photopic luminance. The three fields were the fanning lines of the Hering figure; its converse, the Wundt figure; and the concentric circles of the Orbison figure (Orbison, 1939). Since the Wundt and the Orbison figures have been regarded as inducing illusory effects that are complementary to those of the Hering figure, complementary courses in the development of these illusions may also be expected as viewing conditions are improved.

Apparatus

(a) Stimulus figures. Two horizontal brass bars $18 \times .25 \times .25$ in., painted black, were fixed at their centers, 4 in. apart, on a vertical board $18 \times 18$ in. The ends of these bars could be brought together or made to diverge by means of a worm device operated by $\mathbf{E}$ at the rear of the apparatus. Background lines, .25 in. wide, were printed in black ink on white nonglossy cards, $18 \times 18$ in., which could be inserted behind the horizontal bars on the vertical board. The card for the Hering figure consisted of seven lines, radiating from a central point, to make angles of $20^{\circ}, 40^{\circ}, 60^{\circ}, 90^{\circ}, 60^{\circ}, 40^{\circ}, 20^{\circ}$ with the horizontal bars when these were set horizontally, straight and parallel. In the corresponding card for the Wundt figure, seven lines from a point 2 in. above the center of the upper bar and seven lines from a point 2 in. below the center of the lower bar made the same seven angles of intersection with the parallels and termin- ated midway between them. The card for the Orbison figure had a central black circle of $.5 \mathrm{in}$. diameter and a set of concentric circular bands $.25 \mathrm{in}$. wide and 1 in. apart.

(b) Acuity target. A Snellen "E" of $1.65 \times 1.65$ in., having limb width of $0.33 \mathrm{in}$. was set on a white nonglossy card $18 \times 18$ in. A second acuity target consisted of a similar card with a Snellen " $E$ "' having 0.25 in. limb width.

(c) Display. The stimulus figure was focused on an opal glass screen at the rear of a bellows camera which was set in a partition between two dark rooms. The vertical board could be set at one of five distances in front of the camera by sliding it to marked positions along an $18 \mathrm{ft}$. table. At these fivedistances the images of the stimulus pattern on the opal glass screen were square shapes with sides of $.75,1.0,1.4,2.2$, and 3.9 in. respectively. The luminance of the display to the $S$ was .5 footlamberts.

\section{Subjects and Procedures}

The Ss were 12 University students with at least $20 / 20$ vision and no pronounced astigmatism. They were randomly allocated to an experimental and a control group of six Ss each. They sat in a feebly illuminated room facing the display which, when using a chin rest, was at eye level. Each $S$ sat at a distance from the screen at which he could name the orientation of the larger " $E$ " acuity target with an accuracy of four out of four or seven out of eight correct judgments but was not equally successful with the smaller one. In the display these targets had limb width of .014 and .011 and yielded viewing distances ranging from 59 to 84 in. The three figures and the five sizes of display made 15 combinations; these were presented randomly to each $S$ who was required to say when the lines, adjusted by $\mathbf{E}$ from different prescribed starting positions, became parallel. The six Ss in the control group had essentially the same treatment except that the three background fields were replaced by a non-glossy white card of the same dimensions.

\section{RESULTS AND DISCUSSION}

Each $S$ made six settings of the horizontal bars for each of the three figures at each of the five sizes of the display. These six scores, representing the separation of the ends of the horizontal bars on the vertical board, were averaged to give a score for each $S$ who thus had 15 scores: one for each size-figure combination. Average scores are shown in Fig. 1.

At the three largest sizes of the display the characteristic illusion associated with each background field was obtained, the bars being set more widely apart at 


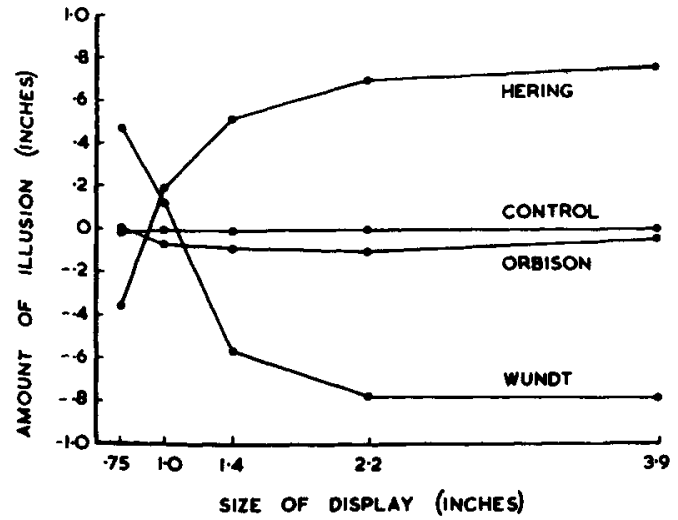

Fig. 1. Amount of illusion as a function of the size of the display. The amount of illusion is the average separation of the bars at the edge of the display less their separation when parallel.

the ends in order to appear parallel in the Hering illusion, but closer together in the case of the other two. The Wundt and Hering illusions developed symmetrically with increments in the size of the display; the Orbison figure yielded a lesser amount of illusion and a different course of development.

These results are more easily interpreted in terms of local influences at the intersections of lines, than in terms of overall configurational effects (Marshall \& Di Lollo, 1963). In the Wundt and Hering figures the apparent displacement of segments of the horizontal lines may be due to an illusion arising from intersection of lines set at acute angles (Titchener, 1910). These local effects are reduced as the intersections become less clearly seen with reduction in the size of the display. In the Orbison figure here used, the number of concentric circles should have been sufficient to generate a field of forces but the angles formed by the horizontal lines and the background concentric circles were considerably greater than the acute angles in the other two figures. The process of overestimation of acute angles was hence less effective.

A reversal of the normal illusion was obtained in the Wundt and Hering figures at the smallest size of the display $(p<.01)$. Some trials suggest that this effect is not obtained when the most slanted radial lines $\left(20^{\circ}\right)$ are removed from the inducing fields. This result supports the interpretation that the negative illusion arises from an assimilation of the end segment of a horizontal line with the end segment of the most slanted radial lines when, at a given level of luminance, the distance between them at the furthest point is below a certain critical value of angle to the eye. Such assimilation may arise either as a result of the acuteness of the angle itself (Adam, 1964) or, as here, as a result of the smallness of the angle subtended to the eye by the relevant section of the figure.

The development of the illusion and the points of transition from negative to normal illusion appear to depend on the smallest size of resolvable detail. Because of the level of luminance employed, the size of minimum resolvable detail at the highest level of impoverishment was intermediate in the present study between that possible under the photopic and scotopic conditions of an earlier experiment (Marshall \& Di Lollo, 1963), and the amount of negative illusion was also intermediate.

\section{References}

Adam, J. A note on visual illusions of direction. Austral. $J$ Psychol., 1964, 16, 53-56.

Marshall, A. J., \& Di Lollo, V. Hering's illusion with impoverishment of the stimulus in scotopic and photopic vision. Amer. $J$ Psychol, 1963, 76, 644-652.

Orbison, $\boldsymbol{W}$. D. Shape as a function of the vector field. Amer. $J$. Psychol., 1939, 52, 31-45.

Titchener, E. B. A textbook of psychology. New York: Macmillan, 1910 . 